\title{
Erratum to: Tunable Nanostructures as Photothermal Theranostic Agents
}

\author{
Joseph K. Young, ${ }^{1}$ Elizabeth R. Figueroa, ${ }^{2}$ and Rebekah A. Drezek ${ }^{1,2}$ \\ ${ }^{1}$ Department of Electrical and Computer Engineering, Rice University, 6100 Main Street, Houston, TX 77005, USA; and \\ ${ }^{2}$ Department of Bioengineering, Rice University, 6100 Main Street, Houston, TX 77005, USA
}

\section{Erratum to: Annals of Biomedical Engineering (2012) 40(2):438-459 DOI 10.1007/s10439-011-0472-5}

Figure captions 1 through 17 and 19 point to the wrong references. The corrected references and author permissions are listed below.

Figure 1. Near-infrared light $(650-900 \mathrm{~nm})$ is of particular interest in biological applications as it is minimally absorbed by biological chromophores and water. Reprinted by permission from Macmillan Publishers Ltd, Weissleder et al. ${ }^{92}$ Copyright 2001.

Figure 2. (a) UV-Vis-NIR absorption spectra of nine HGN samples with varying diameters and wall thicknesses. (b) Image showing the color range of HGN solutions. The vial on the far left contains solid gold nanoparticles, the rest are HNGs with varying diameters and wall thicknesses. Reprinted with permission from Schwartzberg et al. ${ }^{79}$ Copyright 2006 American Chemical Society.

Figure 3. Calcein AM staining indicated that cancerous cells remained viable (evidenced by green fluorescent signal) when exposed to $1 \mathrm{~mW}$ laser power, regardless of nanoparticle presence. At $50 \mathrm{~mW}$ laser output a red fluorescent EthD-1 signal indicative of membrane damage was observed in cells exposed to anti-HER2 functionalized GGS NPs only where the laser was applied. Laser exposure alone was harmless to cells, as was laser exposure combined with nonspecifically targeted nanoparticles. Scale bar $=250 \mu \mathrm{m}$. Reprinted with permission from Day et al. ${ }^{15}$

Figure 4. Kaplan-Meier survival of mice following treatment with GGS NPs and laser irradiation. There is a statistically significant increase in survival with $48 \mathrm{~h}$ accumulation compared to $24 \mathrm{~h}$ accumulation for the GGS NP treated mice and no difference for $48 \mathrm{~h}$ GGS NP treated mice as compared to gold silica

\footnotetext{
Address correspondence to Rebekah A. Drezek, Department of Bioengineering, Rice University, 6100 Main Street, Houston, TX 77005, USA. Electronic mail: jky1@rice.edu, joekyoung@gmail. com, lizfig@rice.edu,drezek@rice.edu

The online version of the original article can be found under doi: 10.1007/s10439-011-0472-5.
}

nanoshell treated mice. Reprinted by permission from Gobin et al. ${ }^{26}$

Figure 5. (a) The extinction $\left(C_{\text {ext }}\right)$, absorption $\left(C_{\text {abs }}\right)$, and scattering $\left(C_{\text {sca }}\right)$ cross-sections (note that $C_{\text {ext }}=$ $C_{\text {abs }}+C_{\text {sca }}$ ) calculated using the DDA method for a gold nanocage of $45 \mathrm{~nm}$ in edge length and $3.5 \mathrm{~nm}$ in wall thickness, and with the geometry depicted in the inset in (d). The alloy composition of the nanocages is $\mathrm{Au}_{3} \mathrm{Ag}$. (b) SEM images of $\mathrm{Ag}$ nanocubes prepared by sulfide-mediated polyol synthesis. The inset shows TEM image of the Ag nanocubes. (c) Normalized Vis-NIR extinction spectra recorded from aqueous suspensions of nanostructures after titrating $\mathrm{Ag}$ nanocubes with different amounts of a $\mathrm{HAuCl}_{4}$ aqueous solution. Note that the spectrum in red is corresponding to the Au nanocages shown in (d). (d) SEM image of $\mathrm{Au}$ nanocages prepared by refluxing an aqueous solution containing both silver nanocubes and $\mathrm{HAuCl}_{4}$. The inset shows a TEM image of the $\mathrm{Au}$ nanocages. Reprinted with permission from Chen et al. ${ }^{10}$ Copyright 2007 American Chemical Society.

Figure 6. SK-BR-3 breast cancer cells that were treated with immuno gold nanocages and then irradiated by $810-\mathrm{nm}$ laser at a power density of $1.5 \mathrm{~W} / \mathrm{cm}^{2}$ for 5 min showed a well-defined circular zone of dead cells as revealed by: (a) calcein AM assay (where green fluorescence indicates the cells were live), and (b) EthD-1 assay (where red fluorescence indicates the cells were dead). In the control experiment, cells irradiated under the same conditions but without immuno gold nanocage treatment maintained viability, as indicated by (c) calcein fluorescence assay, and (d) the lack of intracellular EthD-1 uptake. Modified with permission from Chen et al. ${ }^{10}$ Copyright 2007 American Chemical Society.

Figure 7. (a) Photograph of a tumor-bearing mouse under the photothermal treatment. $100 \mu \mathrm{L}$ of PEGylated nanocages at a concentration of $9 \times 10^{12}$ particles $/ \mathrm{mL}$ or saline was administrated intravenously through the tail vein as indicated by an arrow. After the nanocages had been cleared from the circulation (72 $\mathrm{h}$ after injection), the tumor on the right frank was irradiated by the diode laser at $0.7 \mathrm{~W} / \mathrm{cm}^{2}$ with a beam 
size indicated by the dashed circle. (b-g) Thermographic images of (b-e) nanocage-injected and ( $\mathrm{f}-\mathrm{i})$ saline-injected tumor-bearing mice at different time points: (b, e) $1 \mathrm{~min}$, (c, f) $3 \mathrm{~min},(\mathrm{~d}, \mathrm{~g}) 5 \mathrm{~min}$, and (e, i) 10 min. (j) Plots of average temperature within the tumors (dashed circle) as a function of irradiation time. All scale bars are $1 \mathrm{~cm}$. Reprinted with permission from Chen et al. ${ }^{8}$

Figure 8. F-FDG PET/CT co-registered images of mice intravenously administrated with either saline or Au nanocages, followed by laser treatment: (a) a salineinjected mouse prior to laser irradiation; (b) a nanocage-injected mouse prior to laser irradiation; (c) a saline-injected mouse after laser irradiation; and (d) a nanocage-injected mouse after laser irradiation. The white arrows indicated the tumors that were exposed to the diode laser at a power density of $0.7 \mathrm{~W} / \mathrm{cm}^{2}$ for 10 min. (e) A plot showing the ratios of laser-treated tumor (Rt tumor) to non-treated tumor (Lt tumor) ${ }^{18}$ F-FDG standardized uptake values (SUV, $p<0.001)$. Reprinted with permission from Chen et al. ${ }^{8}$

Figure 9. Carbon nanotubes with high NIR absorbance solubilized in water. (a) Schematic of a Cy3DNA-functionalized SWNT. The drawing is only a graphic presentation and does not represent the precise way DNA binds on SWNTs. (b) UV-Visible spectra of solutions of individual SWNTs functionalized noncovalently by 15 -mer Cy3 labeled-DNA at various nanotube concentrations (top curve, SWNT concentration $\sim 25 \mathrm{mg} / \mathrm{L}$ in $\mathrm{H}_{2} \mathrm{O}$; lower curves correspond to consecutive $3 \%$ reduction in SWNT concentration). The well defined peaks in the UV-Vis spectra suggest lack of large aggregated SWNTs in the solution by removing bundles by centrifugation. (c) Absorbance at $808 \mathrm{~nm}$ vs. SWNT concentration (optical path $=1 \mathrm{~cm}$ ). Solid line is Beer's law fit to obtain molar extinction coefficient of SWNT $\varepsilon=7.9 \times 10^{6} \mathrm{M}^{-1} \mathrm{~cm}^{-1}$. (Inset) A photo of a DNA-functionalized SWNT solution. (d) AFM image of DNA-functionalized individual SWNTs (height of $1-10 \mathrm{~nm}$ ) deposited on a $\mathrm{SiO}_{2}$ substrate (scale bar: $200 \mathrm{~nm}$ ). Reprinted with permission from Shi et al. ${ }^{81}$ Copyright by the National Academy of Sciences.

Figure 10. Selective targeting and killing of cancer cells. (a) Chemical structure of PL-PEG-FA and PLPEG-FITC synthesized by conjugating PL-PEG--NH with FA or FITC, respectively, for solubilizing individual SWNTs. (b) (Upper) Schematic of selective internalization of PL-PEG-FA-SWNTs into folateoverexpressing $\left(\mathrm{FR}^{+}\right)$cells via receptor binding and then NIR 808-nm laser radiation. (Lower) Image showing death of $\mathrm{FR}^{+}$cells with rounded cell morphology after the process in Upper $(808 \mathrm{~nm}$ laser radiation at $1.4 \mathrm{~W} / \mathrm{cm}^{2}$ for $2 \mathrm{~min}$ ). (Inset) Higher magnification image shows details of the killed cells. (c) (Upper) Schematic of no internalization of PL-PEGFA-SWNTs into normal cells without available FRs. (Lower) Image showing normal cells with no internalized SWNTs are unharmed by the same laser radiation condition as in "b". (Inset) Higher magnification image shows a live normal cell in stretched shape. (d) Confocal image of $\mathrm{FR}^{+}$cells after incubation in a solution of SWNTs with two cargoes (PL-PEG-FA and PL-PEG-FITC). The strong green FITC fluorescence inside cells confirms the SWNT uptake with FA and FITC cargoes. (e) The same as d for normal cells without abundant FRs on cell surfaces. There is little green fluorescence inside cells, confirming little uptake of SWNTs with FA and FITC cargoes. Reprinted with permission from Shi et al. ${ }^{81}$ Copyright by the National Academy of Sciences.

Figure 11. In vivo photothermal effects of PEGSWNTs for tumor obliteration. (a) Representative photographs of the mice treated in different groups at various time points after each treatment (I, PEGSWNTs + NIR; II, untreated; III, PBS + NIR; IV, PEG-SWNTs). (b) Four mice after 60 days of photothermal treatments (I) from four independent sets. (c) Time-dependent tumor growth curves of KB tumor cell xenografts. Tumor volumes were measured three times a week after sample treatments. The results are presented as the arithmetic means with standard deviations of tumor volumes in each group $(n=4)$. Only the PEG-SWNTs + NIR treated group (I) shows significant suppression of tumor growth compared with other experimental groups ( $n=4, p<0.05$, twoway ANOVA). Reprinted with permission from Moon et al. ${ }^{64}$ Copyright 2009 American Chemical Society.

Figure 12. (a) Bird's eye view SEM images of $\mathrm{TiO}_{2}$ NTs. $\mathrm{TiO}_{2}$ NTs were formed by anodic etching of Ti thin foils in an electrolyte consisting of $0.3 \mathrm{wt} . \% \mathrm{NH}_{4} \mathrm{~F}$ and 2 vol. $\% \mathrm{H}_{2} \mathrm{O}$ in ethylene glycol at $60 \mathrm{~V}$ for $17 \mathrm{~h}$. (b) TEM image of the $\mathrm{TiO}_{2}$ NT fragments with sizes $<220 \mathrm{~nm}$ prepared by ultrasonication and filtration through a $220 \mathrm{~nm}$ microfilter. Modified with permission from Lee et al..$^{45}$

Figure 13. Comparison of the temperature of $\mathrm{TiO}_{2}$ NTs with those of other inorganic nanomaterials such as Au NPs and SWCNTs which have recently been reported to be potential therapeutic agents for cancer thermotherapy during NIR laser irradiation at illumination intensities of $300 \mathrm{~mW} / \mathrm{cm}^{2}$. TiO 2 NT, Au NP and SWCNT samples are not in a state of suspension but are all in a state of dry solid; in other words, they have equal concentrations $(100 \%)$. Reprinted with permission from Lee et al. ${ }^{45}$

Figure 14. PNB cell theranostic with multi-stage tunable PNB: (a) cell is targeted with NP-antibody conjugates and intracellular NP clusters are formed 
through the receptor-mediated endocytosis, (b) the first (diagnostic) PNB provides the data on a cell and allows one to determine the parameters of the next laser pulse, (c) the second PNB delivers mechanical impact (cell damage though membrane disruption is shown) and this action is guided through the increased optical scattering (red arrows) of the second PNB; the PNB is tuned by varying the fluence of the pump pulse (green arrows). Reprinted with permission from Lukianova-Hleb et al. ${ }^{56}$

Figure 15. Tunability and guidance of the biological effects of PNBs: PNB diameter determines specific biological action and optical signal, while the pump pulse parameters determine the PNB diameter. Reprinted with permission from Lapotko et al. ${ }^{42}$

Figure 16. Scanning electron microscopy images of cancer cells after incubation with gold NPs show their membrane coupling (a) and internalization (b), and the result of the generation of a non-invasive PNB with a lifetime of $25 \pm 5 \mathrm{~ns}$ (c) and an ablative PNB with a lifetime of $300 \pm 42 \mathrm{~ns}$ (d). The insets show images of the whole cells. The cells were fixed immediately after being treated with PNBs. Reprinted with permission from Wagner et al. ${ }^{89}$ Copyright 2010, with permission from Elsevier.

Figure 17. Schematic illustration of multifunctional drug-loaded gold nano-shells for synergistic cancer therapy. The novel nanostructure comprises three key parts for multidimensional therapeutic potentials, namely, (I) an anti-EGFR antibody, Cetuximab (CET), as a targeting moiety and signal- transduction inhibitor, (II) a gold nanoshell that has a photothermal effect due to plasmon resonance upon illumination by an NIR laser, and (III) DOX as a chemotherapeutic agent. These three components, incorporated into PLGA nanoparticles, provide synergistic tumoricidal efficacy. Reprinted with permission from Yang et al. ${ }^{99}$ Advanced Materials, Copyright 2009.

Figure 19. Cell viability after various concentrations of $\mathrm{CuS} \mathrm{NPs}$ and laser intensities. Values present are mean \pm standard deviation from triplicate samples. Reprinted with permission from Li et al. ${ }^{48}$ Copyright 2010, with permission from Future Medicine Ltd. 